\title{
Accurate Virus Identification with Interpretable Raman Signatures by Machine Learning
}

\author{
Jiarong Ye ${ }^{1}$, Yin-Ting Yeh ${ }^{2}$, Yuan Xue ${ }^{1}$, Ziyang Wang ${ }^{3}$, Na Zhang ${ }^{2}$, He Liu ${ }^{2}$, Kunyan Zhang ${ }^{3}$, \\ Zhuohang $\mathrm{Yu}^{2}$, Allison Roder ${ }^{4}$, Nestor Perea Lopez ${ }^{2}$, Lindsey Organtini ${ }^{7}$, Wallace Greene ${ }^{6}$, \\ Susan Hafenstein ${ }^{7,8}$, Huaguang $\mathrm{Lu}^{5}$, Elodie Ghedin ${ }^{4}$, Mauricio Terrones ${ }^{2}$, Shengxi Huang ${ }^{3}$, \\ Sharon Xiaolei Huang ${ }^{1, *}$ \\ 1. College of Information Sciences and Technology, Penn State University, University Park, PA, \\ USA \\ 2. Department of Physics, Penn State University, University Park, PA, USA \\ 3. Department of Electrical and Computer Engineering, Penn State University, University Park, PA, \\ USA \\ 4. National Institute of Allergy and Infectious Diseases, National Institutes of Health, Bethesda, \\ MD, USA \\ 5. Department of Veterinary and Biomedical Sciences, Penn State University, University Park, PA, \\ USA \\ 6. Department of Pathology and Laboratory Medicine, Division of Clinical Pathology, Penn State \\ College of Medicine, Hershey, PA, USA \\ 7. Department of Biochemistry and Molecular Biology, Penn State University, University Park, PA, \\ USA \\ ${ }^{8}$ Department of Medicine, Penn State College of Medicine, Hershey, PA, USA
}

Corresponding Author(s):

Sharon Xiaolei Huang

Email: suh972@psu.edu

Author Contributions: Raman Spectroscopy data analysis, ML algorithm design and implementation, classification and correlation tasks, initial manuscript drafting and supplementary materials preparation were conducted by J. Ye, assisted by Z. Wang and K. Zhang; Y.-T. Yeh, A. Roder, N. Perea Lopez, H. Liu, N. Zhang and Z. Yu contributed to Raman spectra data collection, manuscript drafting and figure design. H. Lu, S. Hafenstein, L. Organtini, W. Green, and E. Ghedin contributed virus samples and helped with paper writing; Y.-T. Yeh, Y. Xue, E. Ghedin, and M. Terrones, S. Huang, and S.X. Huang conceived the initial ideas and helped with paper writing.

Competing Interest Statement: The authors declare that they have no competing financial interests.

\section{Classification: Biophysics and Computational Biology}

Keywords: Raman Spectroscopy, Interpretable Machine Learning, Virus Identification

\section{This PDF file includes:}

Main Text

Figures 1 to 8

\section{Abstract}

Rapid identification of newly emerging or circulating viruses is an important first step toward managing the public health response to potential outbreaks. A portable virus capture device coupled with label-free Raman Spectroscopy holds the promise of fast detection by rapidly obtaining the Raman signature of a virus followed by a machine learning approach applied to recognize the virus based on its Raman spectrum. In this paper, we present a machine learning 
analysis on Raman spectra of human and avian viruses. A Convolutional Neural Network (CNN) classifier specifically designed for spectral data achieves very high accuracy for a variety of virus type or subtype identification tasks. In particular, it achieves $99 \%$ accuracy for classifying influenza virus type $A$ vs. type $B, 96 \%$ accuracy for classifying four subtypes of influenza $A, 95 \%$ accuracy for differentiating enveloped and non-enveloped viruses, and $99 \%$ for differentiating avian coronavirus (infectious bronchitis virus, IBV) from other avian viruses. Furthermore, interpretation of neural net responses in the trained CNN model using a full-gradient algorithm highlights Raman spectral ranges that are most important to virus identification. By correlating ML-selected salient Raman ranges with the signature ranges of known biomolecules and chemical functional groups (e.g. amide, amino acid, carboxylic acid) we verify that our ML model effectively recognizes the Raman signatures of proteins, lipids and other vital functional groups present in different viruses and uses a weighted combination of these signatures to identify viruses. The accurate and interpretable machine learning model developed for Raman virus identification presents promising potential in a real-time virus detection system.

\section{Significance Statement}

A portable micro-fluidic platform for virus capture promises rapid enrichment and label-free optical identification of viruses by Raman spectroscopy. A large Raman dataset collected on a variety of viruses enables the training of machine learning $(\mathrm{ML})$ models capable of highly accurate and sensitive virus identification. The trained ML models can then be integrated with the portable device to provide real-time virus detection and identification capability. We validate this conceptual framework by presenting highly accurate virus type and subtype identification results using a convolutional neural network to classify Raman spectra of viruses.

\section{Main Text}

\section{Introduction}

Viral outbreaks can spread very quickly through various populations and lead to epidemics, and in some cases, pandemics. Seasonal influenza takes an estimated 389,000 lives globally each year (1), and the SRAS-CoV-2 pandemic that began in late 2019 has caused more than 167 million infections and over 3.46 million reported deaths globally (2). These infections also come at a tremendous cost to the global economy and threaten to overwhelm healthcare systems. Therefore, it is critically essential to predict, monitor and control virus infection outbreaks by timely and accurately identifying emerging virus strains.

In the case of an outbreak, rapid detection is often the first step for an effective public health response (3). Polymerase chain reaction (PCR) is often the gold standard to detect viruses as it provides high sensitivity and high specificity. However, the turnaround time of a few hours makes it a limited approach for a rapid response. Rapid tests based on antigen detection have a quick turnaround time of a few minutes, but sensitivity is often low. The ideal set-up for rapid detection of new circulating virus types, subtypes, or antigenic variants to inform surveillance and vaccine development is a platform that employs little pre-processing of the samples and has fast and sensitive detection capabilities.

A handheld device that could be taken into the field or clinics would be extremely powerful and quickly become the standard approach for virus surveillance. The prototype of such a portable device was previously proposed (4), known as VIRRION, based on a micro-fluidic platform containing carbon nanotube (CNT) arrays for label-free capture and enrichment of viruses from clinical samples coupled with an optical detection technology using surface-enhanced Raman spectroscopy that is sensitive to surface proteins and other components of viruses. The input to such a device can be virus cultures, saliva, nasal washes, or even exhaled breath. The output of 
the device is the Raman spectra of captured viruses. A schematic demonstration of the device is shown in Fig. $1 A$. Combining the device with the acquisition of Raman spectra and advanced machine learning models that can classify these spectra to identify the type, subtype, and strain of captured viruses promises an innovative system which can quickly detect, track and monitor viral outbreaks in real-time.

Machine learning (ML) has been successfully applied to Raman spectroscopy analysis in various application scenarios, such as cancer detection (5), mineral recognition (6), and bacteria classification (7). One limitation of current ML-based spectra analysis methods is the lack of transparency in the decision-making process and lack of interpretation of the ML models. Although high accuracy is often reported, the trained ML models, especially those based on deep learning (1), are not transparent and do not provide insight into why and how such accuracy is achieved. In this work, we propose a one-dimensional CNN coupled with an interpretation of neural net responses to identify viruses with high accuracy for distinguishing between virus types and subtypes while informing on the Raman signature ranges that are most important to the CNN model for virus identification.

We further investigate the correlation between ML-selected salient Raman ranges and the Raman signatures of biomolecules such as proteins and lipids $(8,9)$. Various Raman peaks of biomolecules have been interpreted. A previous study has shown that influenza viruses can be identified by Raman signals generated by surface proteins and lipids (10). Another study on SARS-CoV-2 detected peaks corresponding to the spike protein using Raman spectroscopy (11). However, these studies lack quantitative analyses and peak-matching to functional groups (1214). In our work, we focus on gathering information on Raman signatures of protein-related functional groups such as amide, amino acid, carboxylic acid, and the signatures of lipids and lipid-related functional groups such as aliphatic chains from the literature $(8,9,15)$, and our own set of experiments using the receptor binding domain (RBD) of the SARS-CoV-2 spike protein. We find that these known signature ranges correlate well with the key Raman frequency ranges located by our CNN-based ML model. We also design a novel quantifiable metric to measure the level of correlation between the ML-selected ranges and the signature ranges of particular biomolecules.

\section{Results}

In this section, we start with an introduction of the preparation of virus samples and virus Raman spectra, as illustrated in the schematic diagram (Fig. 1A) for the VIRRION platform (4) used to capture viruses and obtain Raman spectra of various viruses. Next, we present the architecture for the 1D CNN classifier used to identify viruses from Raman spectra and compute Raman feature importance values (Fig. 1B). At last, we report and analyze results from our proposed framework, including classification results for virus identification, and findings from correlating important Raman wavenumber ranges determined from ML classification and Raman peak ranges of different biomolecules and/or chemical functional groups. 
Virus Samples Preparation. We conduct machine learning experiments using a dataset consisting of Raman spectra of three groups of viruses, including human respiratory viruses, avian respiratory viruses, and human enteroviruses. The human respiratory viruses were propagated using MDCK cell lines, and the enteroviruses were propagated using HEK 293 cell lines. The avian respiratory viruses were propagated using specific-pathogen-free embryonating chicken eggs. Details of the virus sample preparation procedures can be found in the Virus samples preparation subsection under the Materials and Methods section.

Virus Raman Spectra Data Collection and Pre-processing. The VIRRION platform (4) constructed with nitrogen-doped carbon nanotube arrays and gold ( $\mathrm{Au}$ ) nanoparticles was used as SERS substrate for collecting Raman spectra from virus samples. $100 \mu \mathrm{L}$ of each virus sample was directly dropped (drop-cast) onto the VIRRION Au-CNT substrate and air-dried for $10 \mathrm{hrs}$ prior to Raman measurements. Raman data acquisition was recorded using a Horiba-LabRAM HR Evolution system with a $785 \mathrm{~nm}$ diode laser line. The laser power on the sample was ca. 3.6 $\mathrm{mW}$, focused through a $100 \times$ objective. The $600 \mathrm{gr} / \mathrm{mm}$ grating was used with a spectral range from $500 \mathrm{~cm}^{-1}$ to $2000 \mathrm{~cm}^{-1}$. The typical acquisition time was $30 \mathrm{~s}$.

Fig. 2 shows the numbers of Raman spectra for the Human respiratory viruses, avian viruses, and Human enteroviruses in our dataset. Considering that the numbers of spectra vary among viruses (indicating the presence of data imbalance), we adopted a data augmentation strategy by random oversampling (16). For any classification task, the oversampling augmentation is implemented for virus types with fewer spectra in the training set by bootstrapping, a statistical technique that samples data with replacement (17), so that after the augmentation, the number of spectra of every virus type matches that of the virus type with the largest number of training spectra for the task.

Before feeding the raw Raman spectra as inputs to ML classifiers, we applied the asymmetric least squares smoothing algorithm (18) for baseline correction on each Raman spectrum for all types and subtypes of viruses. In Fig. $3 A$, we show human FLUA and FLUB example spectra before and after baseline correction. For illustration of the spectrum data distribution after baseline correction, we visualize the FLUA and FLUB spectra, using a t-distributed stochastic neighbor embedding (t-SNE) (19) plot (Fig. 3B). In SI Appendix, Fig. S9, we further compare the t-SNE plots before and after baseline correction, for all spectra of all virus types in our entire dataset. From the comparison, we observe that applying baseline correction makes the spectra of different viruses more distinguishable thus makes virus classification tasks easier to achieve high accuracy. More details about the baseline correction algorithm and parameters used for generating the t-SNE plots are explained in the Virus Raman spectra preprocessing subsection under the Materials and Methods section.

Convolutional Neural Network for Virus Identification based on Raman Spectra. To perform virus identification from Raman spectra, we compared the performances of several different machine learning models including XGBoost (20), and CNN. XGBoost is a popular machine learning method similar to the Random Forest (21) method. Instead of an ensemble of multiple decision trees in a random forest, XGBoost uses a boosting style ensemble which iteratively builds more decision trees in the learning process. CNN, in comparison, has stronger feature representation capability. However, widely used 2D convolutional kernels are not appropriate for sequence-like data such as Raman spectra. To this end, we designed a one-dimensional CNN to extract features from Raman spectra and perform accurate virus identification. Fig. $1 B$ demonstrates the architecture of our proposed 1D-CNN for virus classification using spectra. Details about the architecture and training process of our CNN classifier can be found in the CNN architecture and training details subsection under the Materials and Methods section. 
We measured classification performance using three metrics (accuracy, sensitivity, and specificity). Comparing CNN and XGBoost, our 1D-CNN model achieved better performance in all classification tasks, including virus identification from all possible virus types, differentiating enveloped from non-enveloped viruses, classifying different types of Human Respiratory viruses, differentiating Human Flu A from Human Flu B viruses, identification of the subtype of Influenza A viruses, and classification of Avian viruses; Fig. 4 summarizes the classification results. The actual metric numbers for each virus group and all classification experiments are included in $S I$ Appendix, Fig. S1-S4 and S6-S7. Among all virus types and subtypes, the CNN classifier achieved the highest identification accuracies for IBV coronavirus and influenza A virus, around $98 \%$ and $97 \%$, respectively (SI Appendix, Fig. S7).

Interpretation of Raman Signatures of Viruses. While CNN achieves promising classification results, neural networks including CNNs are known to be "black-boxes" and lack interpretability of the decision-making process (22). Recent advances in interpretability of Neural Network models have alleviated this concern by offering numerous ways of visualizing the weights and features within the NN layers (23-28). Here we propose a method of interpreting our 1D CNN decisionmaking process by calculating a "feature importance map", which shows the relative importance of wavenumbers in recognizing the corresponding virus types of input spectra. The wavenumbers (or features) with the highest importance values can tell us what Raman spectroscopy detects from the viruses that machine learning employs to identify them. The calculation of the feature importance map is based on a Full-gradients algorithm (29), as illustrated in the overview diagram Fig. $1 B$ and detailed in the Calculation of Raman feature importance maps using CNN neural network responses subsection under the Materials and Methods section.

The feature importance map allows us to identify Raman signature ranges deemed most important by the CNN classifier for virus identification. We can then correlate these ML-selected salient Raman ranges with the signature peak ranges of known biomolecules and chemical functional groups such as lipids, proteins, nucleic acids, amino acids and amide, to seek insights about what differences in biomolecular composition among viruses are captured in the Raman spectra and then used by $M L$ to recognize viruses.

To measure the level of correlation between the ML-selected important wavenumer ranges and Raman peak wavenumber ranges of a known biomolecule, we propose a quantifiable metric termed "matching score". It is a ratio with the numerator as the number of overlapped wavenumbers between ML-determined important ranges and Raman peak ranges of the biomolecule, and the denominator as the total number of wavenumbers in the Raman peak ranges of that biomolecule (Fig. 5). The higher the matching score, the more likely the signatures of the biomolecule contribute substantially to distinguish viruses. Using this quantifiable metric, we can make some educated guesses about the relative importance of biomolecules in virus identification tasks.

In Figs. 6-8 we show example feature importance maps from CNN and their correlation with Raman peak ranges of biomolecules known to exist in viruses. In choosing which biomolecules and functional groups to evaluate, we used prior knowledge about the composition of the RNA viruses in our study. Some viruses are enveloped (FLUA and FLUB, IBV Coronavirus, RSV), some are not (Reovirus, Enterovirus CVB1/CVB3/EV70/EV71/PV2, Rhino), thus, we included lipid as one type of biomolecular to evaluate. We also included surface protein-related functional groups and individual amino acids, such as Amide, phenylalanine and tyrosine. Nucleic acid RNA is also included. Details about how we obtained the Raman peak ranges for the biomolecules and functional groups under consideration are available in the Interpretable Raman signatures subsection under the Materials and Methods section. Next, we present some findings from 
calculating the matching scores between ML-selected important Raman ranges and biomolecule peak ranges for various virus classification tasks.

Enveloped vs. Non-enveloped Virus Classification. We did an experiment to train an ML model to classify enveloped vs. non-enveloped viruses and achieved very high accuracy $(94.8 \%$ accuracy; see SI Appendix, Fig. S6). This ML model could be used for fast screening, to identify whether a new virus is enveloped or non-enveloped. In Fig. 6, we show the Raman feature importance map calculated by this ML model as well as matching scores between ML-selected important ranges and biomolecular peak ranges. From the matching score table, one can see that for this task lipid is shown to be much more important (matching score 51.98\%) than protein-related functional groups (matching scores $25 \%$ and $7.98 \%$ for Amide I and Amide III, respectively). This is consistent with the difference between enveloped and non-enveloped viruses, which is that enveloped viruses have an enclosing phospholipid bilayer whereas non-enveloped viruses do not have the phospholipid bilayer. It is highly likely that the ML model is picking up the signature ranges of lipid to differentiate enveloped from non-enveloped viruses.

Comparison of Classification Tasks that Differentiate Various Flu Types. We trained several ML models to differentiate various Flu types such as avian FLUA from human FLUA, and human FLUA from human FLUB (Fig. 7 and SI Appendix, Fig. S5). From the matching score table, we noted that the Amide III range is not important for classifying avian FLUA vs. human FLUA (matching score 13.16\%) but more important when differentiating human FLUA from human FLUB (matching score 73.68\%). Lipid is more important when differentiating avian FLUA from human FLUA but less important when differentiating human FLUA from human FLUB, likely indicating that the ML model trained for classifying avian FLUA vs. human FLUA is capturing their differences in the envelopes since the phospholipid bilayer of the human viruses comes from a different cell line than the avian viruses that were isolated in eggs. Also, the RNA matching score stands out to be higher (60\%) when differentiating human FLUA from human FLUB, compared to classifying avian FLUA vs. human FLUA (40\%).

Classification of Avian Viruses including IBV Coronavirus. We trained an ML model to differentiate three types of avian viruses and achieved very high accuracy (99.8\%) in identifying the IBV coronavirus (Fig. 8, and SI Appendix, Fig. S1). This shows that the Raman spectra of coronavirus have specific signatures that make them easily identifiable and the proposed technique combining Raman spectroscopy and machine learning can potentially be used for highly reliable detection and identification of coronaviruses. From the matching score table in Fig. 8 , one can see that both lipid and protein peak ranges have high correlation with ML-selected important Raman ranges for this classification task.

\section{Discussion}

Additional Observations about Correlation between ML-selected Important Raman Ranges and Biomolecule Peak Ranges. Comparing the matching scores for the experiment classifying different human respiratory viruses (See SI Appendix, Fig. S6) and the experiment classifying different subtypes of Influenza (FLU) A (See SI Appendix, Fig. S5), we observe that: (1) the relative importance of lipid is higher in the FLU A subtype identification task. (2) There is a significant difference in the relative importance of the Amide III range. While Amide III is very important in respiratory virus classification, it is minimally important in FLUA subtype identification, which could indicate that the spectra of all subtypes of FLUA are very similar in the Amide III range. Amide III is a signature Raman band in proteins, and such a difference of its matching scores indicates that the viruses have different surface proteins-which is indeed the case when comparing different families of viruses-and that the subtypes have smaller changes in their surface proteins-which again is the case since FLUA viruses have Hemagglutinin (HA) and Neuraminidase (NA) on their surface. This is consistent with our preliminary findings about the viruses under study. (3) The two chosen amino acids (phenylalanine and tyrosine) are 
consistently important in respiratory virus type or subtype classification. And, finally, (4) the RNA genome is also generally important and this also matches with the existing knowledge in virology.

Summary and Future Research Directions. In summary, we present results on applying machine learning to identify viruses trapped by a portable virus capturing device and imaged by Raman spectroscopy. Our machine learning system based on a CNN implementation shows high accuracy in classifying different types of human and avian viruses. It can also differentiate subtypes of influenza viruses. The interpretation of the neural network responses also provides valuable information about Raman wavenumber ranges that correlate well with the signature ranges of known biomolecules and chemical functional groups existing in viruses. The major contributions of our work are:

(1) We develop a 1D-CNN classifier that achieved high accuracy in multiple virus identification and classification tasks including differentiating Enveloped from Nonenveloped viruses, identifying types of Human Respiratory viruses, differentiating Human Flu A from Human Flu B viruses, classifying subtypes of Influenza A viruses, and differentiating among types of Avian viruses;

(2) We further investigate the association between classifier-selected important Raman ranges and peak ranges of lipids, proteins and relevant chemical functional groups, and observe correlations that are supported by prior knowledge;

(3) Our delivery of promising virus classification results indicates that Raman spectra of different virus types and subtypes contain recognizable signatures that can be identified by machine learning models, which unravels the potential of using interpretable machine learning in a real-time virus surveillance system.

In our future work, we will collect more Raman spectra of different virus samples to build a large virus spectra database for training robust and highly accurate machine learning models. We will study virus evolution and predict antigenic variants using temporal machine learning models trained on Raman spectra of virus strains of different types, from different years and locations. And we will develop better Raman enhancement substrates with better signal strengths and lower noise levels, considering the feedback from ML classification and feature importance identification.

\section{Materials and Methods}

Virus Samples Preparation. Avian influenza virus (AIV) was propagated in specific-pathogenfree (SPF) embryonating chicken eggs (ECE) via allantoic cavity route inoculation at 9-11 days of age. The inoculated ECEs were incubated in a $37^{\circ} \mathrm{C}$ egg incubator for $3 \mathrm{~d}$ (or $72 \mathrm{~h}$ ) and then were removed/chilled at $4{ }^{\circ} \mathrm{C}$ for a minimum of $4 \mathrm{~h}$ or overnight. Allantoic fluid (AF) containing the virus was harvested from each egg using sterile technique (a $3 \mathrm{~mL}$ sterile syringe with a $25 \mathrm{G} \times 5 / 8$ " needle). The harvested AF was clarified by centrifugation at $8000-1000 \mathrm{rpm}$ for $10 \mathrm{~min}$ and then was tested the virus hemagglutination (HA) titers by $\mathrm{HA}$ test. The virus was also measured in embryo infectious doses $50 \%\left(E I D_{50}\right)$ titers by the Reed-Muench method (30). Briefly, the $E I D_{50}$ test was conducted in ECE. The propagated fresh stock H5N2 AIV was prepared in 10-fold serial dilutions from $10^{-1}$ through $10^{-9}$. Each dilution was inoculated into 5 eggs, $0.1 \mathrm{~mL}$ per egg. The inoculated eggs were incubated at $37^{\circ} \mathrm{C}$ for 72 hours. The eggs were candled daily to remove dead eggs to chill them at $4{ }^{\circ} \mathrm{C}$ refrigerator. After 72 hours of incubation, allantoic fluid was harvested from each egg (31).

H1N1, H3N1, FluB, Rhinovirus, and RSV were prepared in Madin-Darby canine kidney-London (MDCK-London) cell culture. MDCK-London cells were cultured in Dulbecco's modified Eagle's medium (DMEM; Invitrogen, Carlsbad, CA) containing 10\% fetal bovine serum and $1 \%$ penicillinstreptomycin, and incubated at $37^{\circ} \mathrm{C}$ in a humidified $\mathrm{CO}_{2}$ incubator. 
Enteroviruses (CVB1, CVB3, EV70, EV71, or PV2) were propagated using HEK 293 cell lines. Infection of cells with enterovirus inoculum, harvesting of cells and media, and additional virus sample preparation steps are documented in the Virus Propagation and Purification subsection in (32).

Virus Raman Spectra Preprocessing. Before feeding the Raman spectra into classifiers as input, it is essential to employ a few preprocessing steps to reduce noise in the spectra that could potentially undermine the classification performance of trained ML models. First, we apply baseline correction with asymmetric least squares smoothing to reduce background noises. This method estimates a polynomial baseline to correct baseline shift in Raman measurements. Then, we adjust the intensities that vary across the spectra of different virus types to a universal scale by normalization. In this step, we apply $L_{2}$ normalization that converts the input vectors to unit vectors. The normalization makes intensities comparable across spectra and facilitates convergence during $\mathrm{ML}$ model training.

For generating the t-SNE plots of spectra data (Fig. 3 and SI Appendix, Fig. S9), we use the scikit-learn (33) machine learning package to perform the t-SNE dimensionality reduction and map high-dimensional data points to a two-dimensional space. We use the default parameters of the package except changing the perplexity value to 50 and the learning rate to 200 . Under this setting of parameters, the data points in the two-dimensional map for FLUA and FLUB spectra fall into clearly distinct clusters, which indicates that a deep learning network capable of nonlinear functional mapping should be able to achieve highly accurate classification on the dataset.

CNN Architecture and Training Details. As shown in Fig. 1B, the Convolutional Neural Network for our task is built with 4 convolutional blocks. Considering the dimension of our training set is $\mathrm{N} \times 1 \times \mathrm{D}_{\mathrm{w}}$, where $\mathrm{N}$ refers to the number of Raman spectra samples in the training set and $\mathrm{D}_{\mathrm{w}}$ is the dimension of Raman wavenumber range, a reasonable option for the convolutional blocks is to adopt 1D CNN layer. Followed by the convolutional layer is a 1D batch normalization layer and an activation layer, in this case we choose ReLU. The kernel size and stride of the 1D CNN layer of the first convolutional block are both set as 1 , with the width fixed while increasing the depth from the input dimension 1 to the dimension of the hidden state, which will be specified later along with other hyper-parameters. Next, for the other three convolutional blocks, kernel size is increased to 3 and stride is set as 2 for reducing the dimension of feature maps by half each time. Followed by the activation layer of the second and the third convolutional blocks, 2 dropout layers with rate 0.5 and 0.25 are applied, respectively, for alleviating over-fitting to the training set. After all convolutional blocks, the last layer for obtaining the final classification results is a fully connected layer with output dimension as $\mathrm{N} \times 1 \times \mathrm{D}_{\mathrm{c}}$, here $\mathrm{D}_{\mathrm{c}}$ is denoted as the number of virus types or subtypes, depending on the classification task and specific dataset used for that task.

During training, we apply a 5-fold cross-validation and stratified sampling for each fold based on the virus types (or subtypes) to ensure that after splitting the dataset into training and testing sets, every type (or subtype) gets equal representation in both sets regardless of how unbalanced the data distribution is. For fair comparison, we run the cross-validation for 5 times and obtain the average score for all metrics across the 5 test runs. All hyper-parameters are fixed for each run, the learning rate is set as 0.001 and trained for 1000 epochs with hidden dimension set to 128 for the first convolution block, and then decreases by half for every subsequent convolutional block.

\section{Calculation of Raman Feature Importance Map using CNN Neural Network Responses.}

While the CNN classifiers trained for virus identification tasks achieve high performance, we are interested in learning what Raman features are utilized by these classifiers to differentiate among viruses. To this end, we propose an algorithm for the CNN to infer the feature importance value for each specific wavenumber for further investigation of interpretability. With regards to the interpretation of feature extraction and selection by neural networks, saliency map has been widely considered as an intuitive and well-established method to visualize the importance value for each unit within the input data (23-28). However, in our case, the contribution each wavenumber has to the final virus type (or subtype) classification is highly unlikely to be 
independent from each other. A more reasonable assumption is that the distinguishable features from Raman spectra of a specific virus type (or subtype) are composed of a set of Raman signature ranges, besides individual wavenumbers of Raman spectra. Hence a desirable design of saliency map representation for interpretation is expected to include both attributions to ensure the completeness of the feature map. By leveraging features from input vector and neurons from intermediate layers simultaneously, the full-gradient algorithm (29) is proven to a sensible representation of CNN interpretability in terms of the capability to capture both local and global attributions from each Raman spectra wavenumber and signature ranges. As the full gradient representation for neural network visualization (29) was originally designed and applied on natural images, we adapt and modify the full-gradient algorithm to accommodate the 1D vector inputs, as the format of Raman spectra is in our case. As shown in Fig. 1B, the process for extracting feature importance for each virus type or subtype is demonstrated below the architecture of CNN. The full gradient feature importance map extracted is defined as

$$
S_{f}(x)=\psi\left(\nabla_{x} f(x) \odot x\right)+\sum_{l \in L} \sum_{c \in c_{l}} \psi\left(\nabla_{b} f(x, b)_{c} \odot b\right) .
$$

Here, the saliency map of the full-gradient representation consists of two parts---input gradient that is specific to each wavenumber of Raman spectra in the training set, and bias-gradient from each convolutional block. The components of each convolutional block are illustrated in Fig. $1 B$. The approximate network-wide representation of the feature map is considered comprehensive for capturing what the model learned throughout the process of the classification task from both lower and higher levels of abstraction. $c$ refers to the virus type or subtype, depending on the target for a particular classification task. Gradients specific for each $c$ are extracted separately in order to get insights of Raman frequency significance for different types and subtypes of viruses. This process is implemented by activating the virus type (subtype) of interest during backpropagation through the entire set of convolutional blocks while obtaining the cross-entropy loss for each $c . \psi(\cdot)$ refers to the post-processing steps that can be denoted as $\psi(\cdot)=$ bilinearUpsample(normalize $(\operatorname{abs}(\cdot))$. First, the operation that is applicable for both input and bias gradients is the step of obtaining the absolute value of either positive or negative importance to visualize the significance while neglecting the sign. Next, the absolute values of gradients are normalized to the range of $[0,1]$ to optimize the visualization by creating proper viewing contrast. And then for the gradients extracted from convolutional blocks with dimension downsized to different scales of hidden states, we facilitate the aggregation of the block-wise feature maps by up-sampling each to the same dimension as the input vector with bilinear interpolation. The feature map extracted for each virus type or subtype is shown in the format of the area chart in Fig. $1 B$.

Interpretable Raman Signatures. Considering that one of our goals is to make an educated guess as to which biomolecules are more likely to have a significant contribution in differentiating virus types or subtypes, we analyze the correlation between the important Raman ranges from CNN feature importance map and the Raman peak ranges of biomolecules existing in viruses. First, the Raman peaks of biomolecules including lipids, proteins, nucleic acids and proteinrelated chemical functional groups are gathered from the literature $(8,9,15)$ (see SI Appendix, Fig. S8 for detailed peak ranges). We note that for a specific functional group, the specific Raman range can vary when measured in different environments. Here we included all the possible Raman ranges for the generality of our analysis. For RBD proteins and amino acids (Tyrosine, Phenylalanine), we measured their Raman spectra in our own experiments and then located the peaks of the Raman spectra by adopting the python package (34). A shift of 5 wavenumbers is granted to each peak to construct the peak ranges for each biomolecule (i.e. as a range for each peak). Second, given a Raman feature (i.e. wavenumber) importance map calculated using the full-gradient algorithm for CNN, we extract important Raman ranges by applying a threshold on the calculated feature importance values. We apply a Savitzky-Golay filter (35) on the relatively noisy importance values, with the length of the filter window set as 17. 
Then, a 40-percentile threshold is applied to extract ranges in the feature map that consist of wavenumbers with corresponding importance values above the threshold. Finally, the quantifiable metric---the matching score as demonstrated in Fig. 5---is used to measure the level of correlation between Raman peak ranges of biomolecules and important Raman ranges identified by ML. The matching score metric is developed in the format of a ratio, where the numerator of the ratio is the amount of overlap (i.e. number of overlapped wavenumbers) between the MLcalculated important Raman ranges for identifying a particular virus type and the Raman peak ranges of a certain biomolecule, and the denominator is the total number of wavenumbers in the biomolecule's peak ranges. Thus, the matching scores are in the range of $[0,1]$ : a matching score of 1.0 means that the biomolecule's entire peak ranges are considered important by the CNN classifier for identifying the virus; a matching score of 0 indicates no wavenumber within the biomolecule's peak ranges is considered important by the classifier; and when the matching score value is between 0 and 1 , the higher the score, the more likely that the biomolecule is important for identifying that particular type of virus. We report the matching scores for all our $\mathrm{ML}$ classification tasks in SI Appendix, Fig. S1-S6, and show the Raman peak ranges for biomolecules in Fig. S8.

\section{Acknowledgments}

We thank the National Science Foundation's Growing Convergence Research Big Idea (under Grant OIA-1934977), and National Science Foundation's EArly-concept Grants for Exploratory Research (under Grant OIA-2030857).

\section{References}

1. J. Paget, et al., Global mortality associated with seasonal influenza epidemics: New burden estimates and predictors from the GLaMOR Project. J. Glob. Health 9, 020421 (2019).

2. W. H. Organization, Others, Coronavirus disease 2019 (COVID-19): situation report, 82 (2020).

3. F. Keesing, et al., Impacts of biodiversity on the emergence and transmission of infectious diseases. Nature 468, 647-652 (2010).

4. Y.-T. Yeh, et al., A rapid and label-free platform for virus capture and identification from clinical samples. Proc. Natl. Acad. Sci. U. S. A. 117, 895-901 (2020).

5. S. Li, et al., Noninvasive prostate cancer screening based on serum surface-enhanced Raman spectroscopy and support vector machine. Appl. Phys. Lett. 105, 091104 (2014).

6. S. T. Ishikawa, V. C. Gulick, An automated mineral classifier using Raman spectra. Comput. Geosci. 54, 259-268 (2013).

7. A. Walter, et al., From bulk to single-cell classification of the filamentous growing Streptomyces bacteria by means of Raman spectroscopy. Appl. Spectrosc. 65, 1116-1125 (2011).

8. K. Czamara, et al., Raman spectroscopy of lipids: a review. J. Raman Spectrosc. 46, 4-20 (2015).

9. D. Němeček, G. J. Thomas Jr, "Raman spectroscopy of viruses and viral proteins" in Frontiers of Molecular Spectroscopy, (Elsevier, 2009), pp. 553-595.

10. J.-Y. Lim, et al., Identification of newly emerging influenza viruses by detecting the virally infected cells based on surface enhanced Raman spectroscopy and principal component analysis. Anal. Chem. 91, 5677-5684 (2019). 
11. D. Zhang, et al., Ultra-fast and onsite interrogation of Severe Acute Respiratory Syndrome Coronavirus 2 (SARS-CoV-2) in environmental specimens via surface enhanced Raman scattering (SERS). bioRxiv (2020) https:/doi.org/10.1101/2020.05.02.20086876.

12. Y. Liu, et al., Label and label-free based surface-enhanced Raman scattering for pathogen bacteria detection: A review. Biosens. Bioelectron. 94, 131-140 (2017).

13. M. Reyes, et al., Exploiting the anti-aggregation of gold nanostars for rapid detection of hand, foot, and mouth disease causing Enterovirus 71 using surface-enhanced Raman spectroscopy. Anal. Chem. 89, 5373-5381 (2017).

14. K. Moor, et al., Noninvasive and label-free determination of virus infected cells by Raman spectroscopy. J. Biomed. Opt. 19, 067003 (2014).

15. Y. H. Ong, M. Lim, Q. Liu, Comparison of principal component analysis and biochemical component analysis in Raman spectroscopy for the discrimination of apoptosis and necrosis in K562 leukemia cells. Opt. Express 20, 22158-22171 (2012).

16. C. Shorten, T. M. Khoshgoftaar, A survey on image data augmentation for deep learning. J. Big Data 6 (2019).

17. G. James, D. Witten, T. Hastie, R. Tibshirani, An Introduction to Statistical Learning: with Applications in R (Springer, New York, NY, 2013).

18. S.-J. Baek, A. Park, Y.-J. Ahn, J. Choo, Baseline correction using asymmetrically reweighted penalized least squares smoothing. Analyst 140, 250-257 (2015).

19. L. van der Maaten, Visualizing Data using t-SNE (2008) (May 23, 2021).

20. T. Chen, C. Guestrin, XGBoost in Proceedings of the 22nd ACM SIGKDD International Conference on Knowledge Discovery and Data Mining - KDD '16, (ACM Press, 2016) https:/doi.org/10.1145/2939672.2939785.

21. L. Breiman, Random Forests. Mach. Learn. 45, 5-32 (2001).

22. J. M. Benitez, J. L. Castro, I. Requena, Are artificial neural networks black boxes? IEEE Trans. Neural Netw. 8, 1156-1164 (1997).

23. M. D. Zeiler, R. Fergus, "Visualizing and understanding convolutional networks" in Computer Vision - ECCV 2014, Lecture notes in computer science., (Springer International Publishing, 2014), pp. 818-833.

24. K. Simonyan, A. Vedaldi, A. Zisserman, Deep inside Convolutional Networks: Visualising image classification models and saliency maps. arXiv [Cs.CV] (2013).

25. J. Yosinski, J. Clune, A. Nguyen, T. Fuchs, H. Lipson, Understanding neural networks through deep visualization. arXiv [Cs.CV] (2015).

26. R. R. Selvaraju, et al., Grad-CAM: Visual explanations from deep networks via gradientbased localization in 2017 IEEE International Conference on Computer Vision (ICCV), (IEEE, 2017) https:/doi.org/10.1109/iccv.2017.74.

27. P.-J. Kindermans, et al., "The (Un)reliability of saliency methods" in Explainable Al: Interpreting, Explaining and Visualizing Deep Learning, Lecture notes in computer science., (Springer International Publishing, 2019), pp. 267-280.

28. K. Zolna, K. J. Geras, K. Cho, Classifier-agnostic saliency map extraction. Comput. Vis. Image Underst. 196, 102969 (2020). 
29. S. Srinivas, F. Fleuret, Full-Gradient Representation for Neural Network Visualization in NeurIPS, (2019).

30. G. J. Ebrahim, Virology: principles and applications J. Carter, V. Saunders (eds). J. Trop. Pediatr. 55, 66-66 (2007).

31. S. Zheng, et al., Sizable tunable enrichment platform for capturing nano particles in a fluid. US Patent (2020) (May 30, 2021).

32. K. L. Shingler, et al., The enterovirus 71 A-particle forms a gateway to allow genome release: a cryoEM study of picornavirus uncoating. PLoS Pathog. 9, e1003240 (2013).

33. F. Pedregosa, et al., Scikit-learn: Machine learning in Python. the Journal of machine Learning research 12, 2825-2830 (2011).

34. P. Virtanen, et al., SciPy 1.0: fundamental algorithms for scientific computing in Python. Nat. Methods 17, 261-272 (2020).

35. William H. Press, S. A. Teukolsky, Savitzky-Golay Smoothing Filters. Comput. Phys. 4, 669 (1990). 
Figures and Tables

A
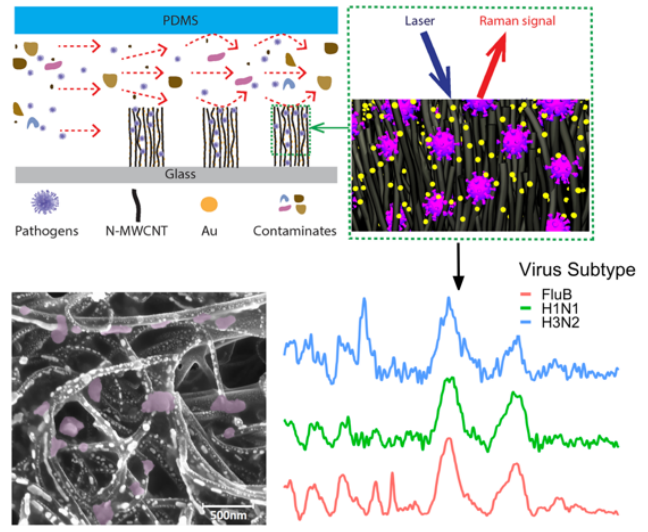

B

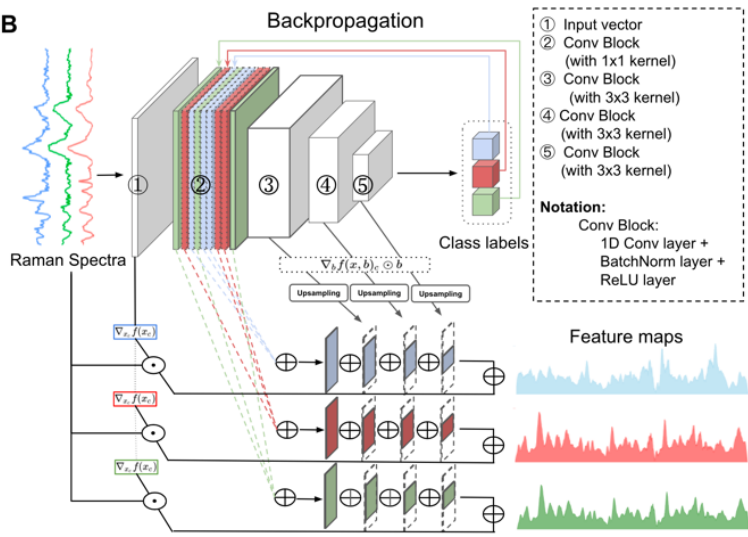

Figure 1. A. Schematics showing the carbon nanotubes (CNTs) device encapsulated in PDMS (polydimethylsiloxane) used to enrich viruses (top left). The viruses are enriched between CNTs where the Au nanoparticles are pre-deposited. Raman spectra are then collected from the virusenriched samples (top right). A Scanning Electron Microscope (SEM) image (bottom left) of a sample shows CNTs, Au nanoparticles, and trapped viruses (purple colored). Raman spectra from different virus samples are shown (bottom right; Flu B in red, H1N1 in green and H3N2 in blue). B. The Convolutional Neural Network (CNN) architecture for virus identification and the process of extracting Raman feature maps that show important Raman signature ranges. The feature maps extracted are class-specific, demonstrating the significant Raman ranges for identifying different virus types (or subtypes, depending on the classification task) in different colors. 


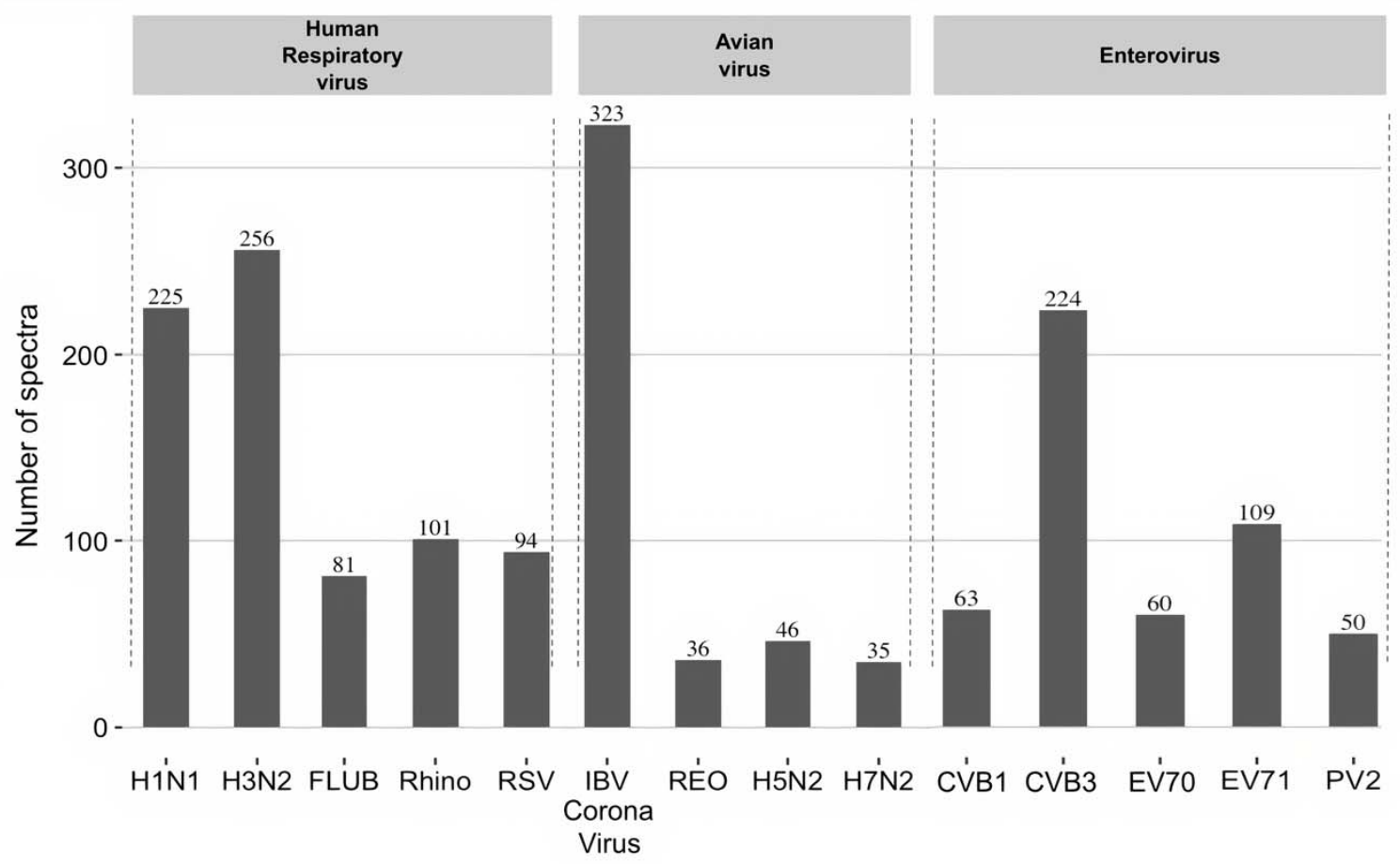

Figure 2. Number of spectra in our dataset for Human respiratory viruses, avian viruses, and Human enteroviruses. H1N1, H3N2, H5N2, and H7N2 are subtypes of the influenza A virus; FLUB: influenza B virus; Rhino: rhinovirus; RSV: respiratory syncytial virus; IBV: infectious bronchitis virus; Reo: reovirus; CVB1 and CVB3: Coxsackievirus B type 1 and 3; EV70 and EV71: enteroviruses. Numbers above each column indicate the number of spectra collected for each virus. 
A
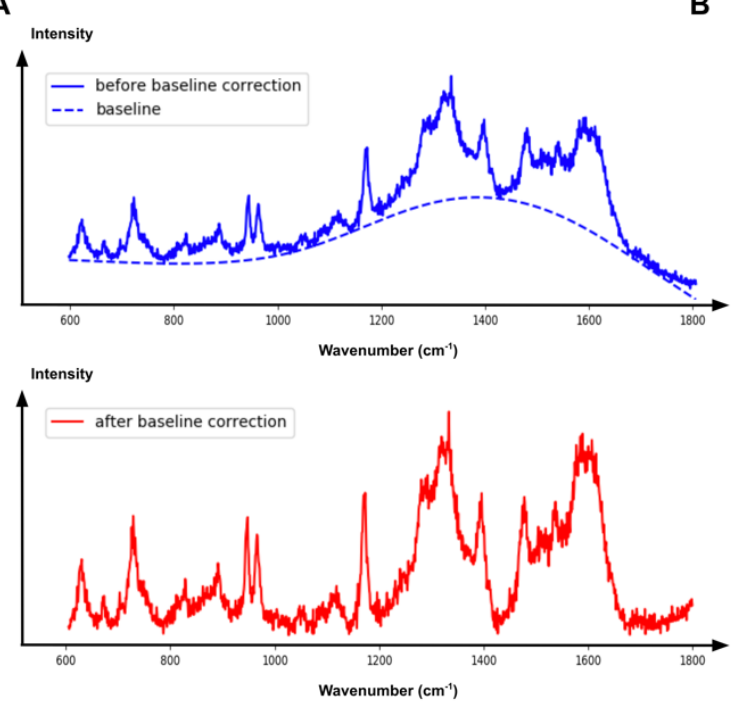

B
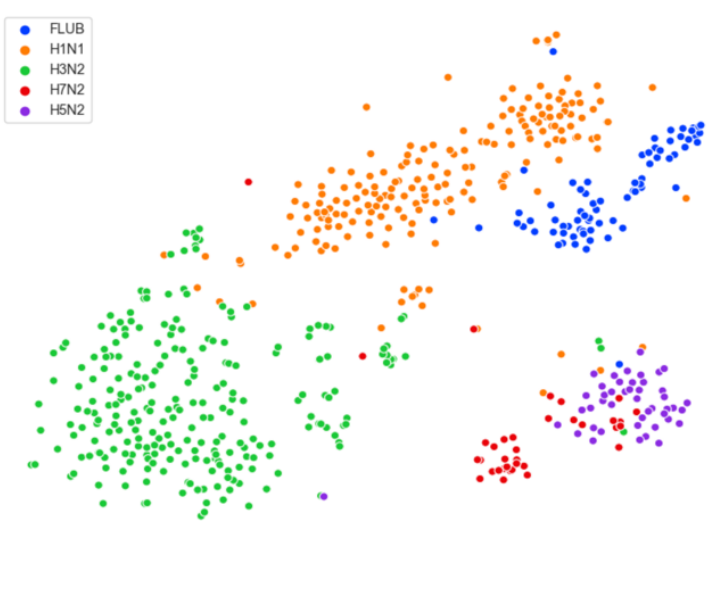

Figure 3. A. Sample Raman spectra before and after baseline correction; B. T-SNE plot of FLUA subtypes (H1N1, H3N2, H5N2, H7N2) and FLUB after baseline correction. 
A

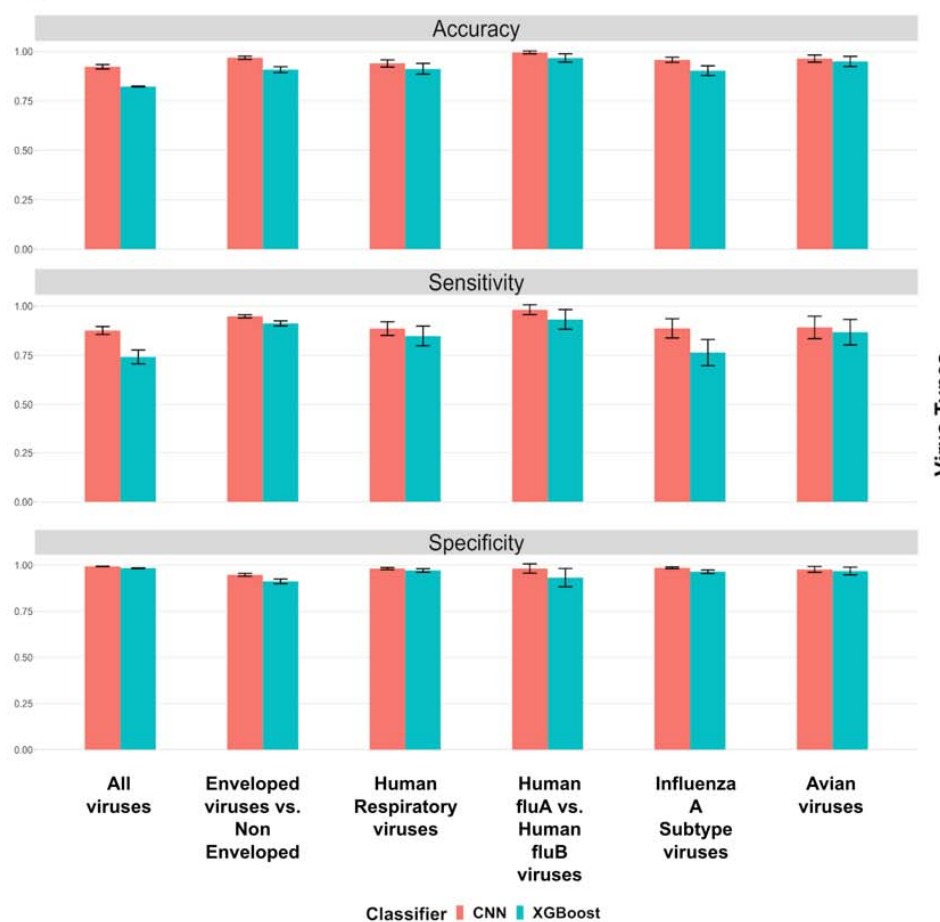

B

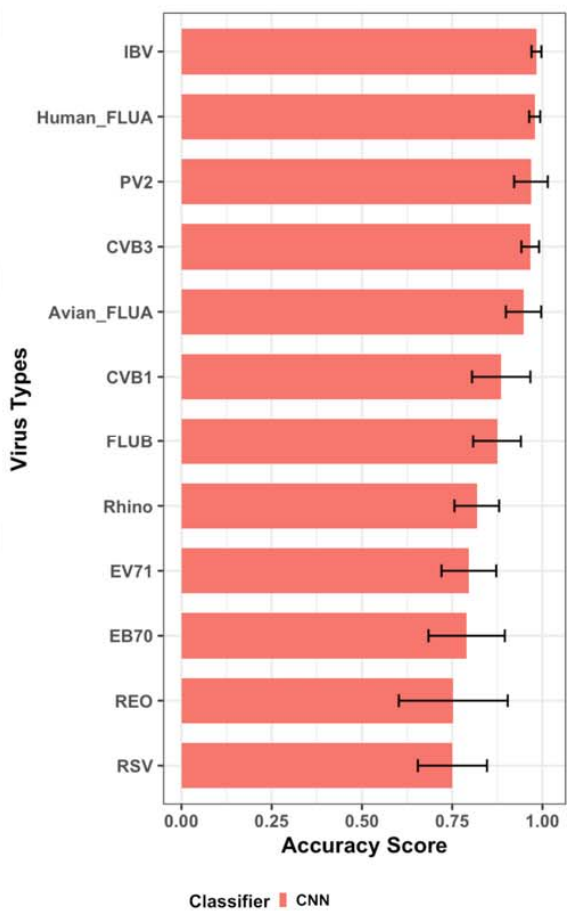

Figure 4. A. The classification performance of our CNN model and the XGBoost model on 6 experiments: 1) All viruses (classification of all virus types): Avian, Enteroviruses, Human Respiratory viruses; 2) Enveloped viruses vs. Non-Enveloped: FLUA and FLUB, IBV Coronavirus, RSV are Enveloped, Reovirus, Enterovirus CVB1/CVB3/EV70/EV71/PV2, Rhino are NonEnveloped; 3) Human Respiratory viruses; 4) Human FLUA vs. Human FLUB viruses; 5) Influenza A subtypes; and 6) Avian viruses. Three metrics (accuracy, sensitivity, and specificity) are measured for both classification models. Results for all metrics are obtained by running a 5fold cross-validation for 5 times for fair comparison (each error bar represents the standard deviation of the corresponding metric score for each experiment across 5 -fold cross-validation in 5 tests); B. Accuracy score for every virus type in the All-viruses classification task (each error bar represents the standard deviation of the corresponding accuracy score for each virus type across 5 -fold cross-validation in 5 tests). 


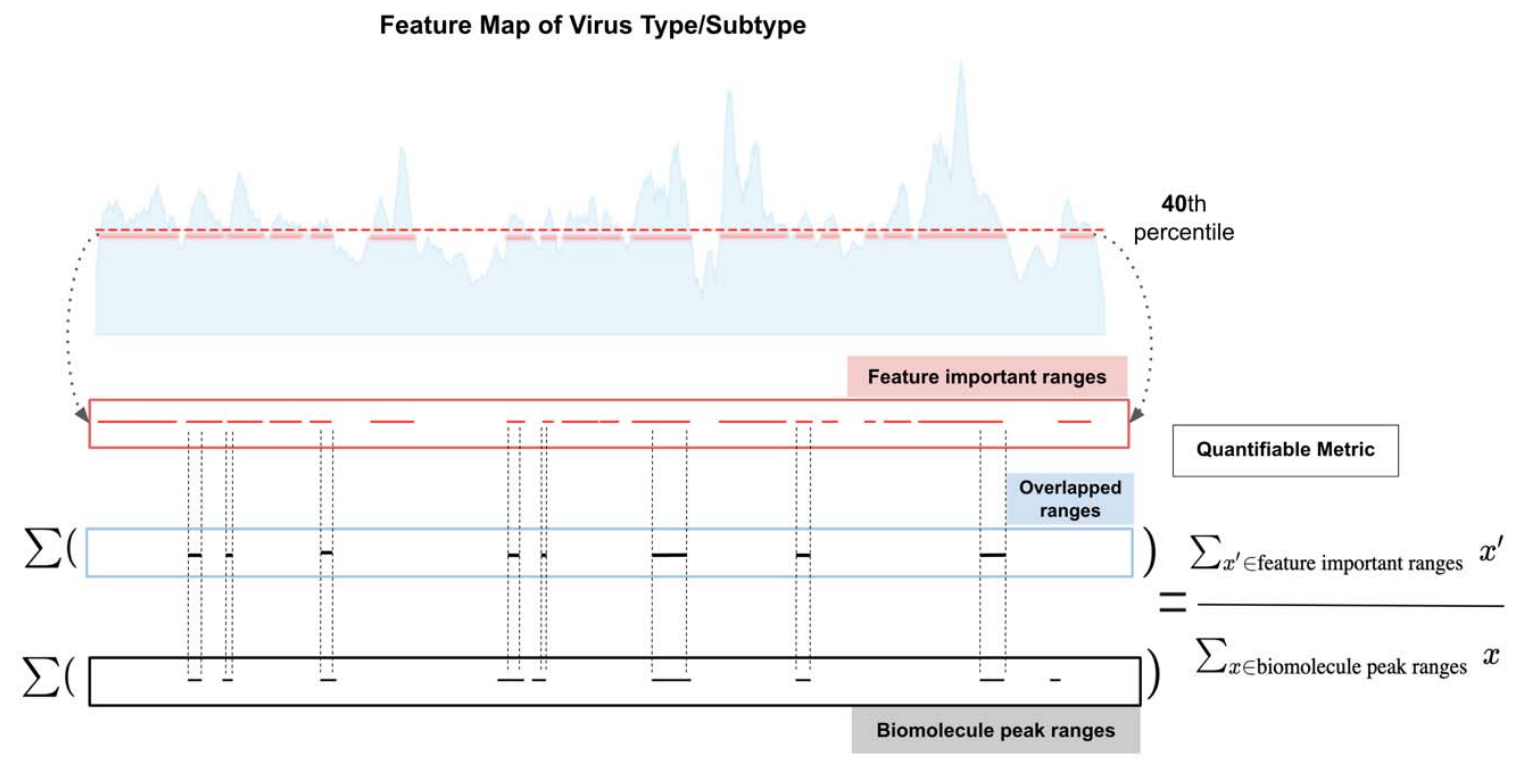

Figure 5. Illustration of the quantifiable matching score calculation leveraging biomolecule peak ranges and important ranges extracted from ML-calculated feature maps of each virus type (or subtype, depending on the classification task). A threshold of 40th percentile is applied to the MLcalculated feature importance map so that Raman bands with importance scores below the threshold are discarded, and the remaining wavenumbers above the threshold are considered as important Raman ranges for identifying the virus based on $\mathrm{ML}$ and can then be correlated with biomolecule peak ranges. 


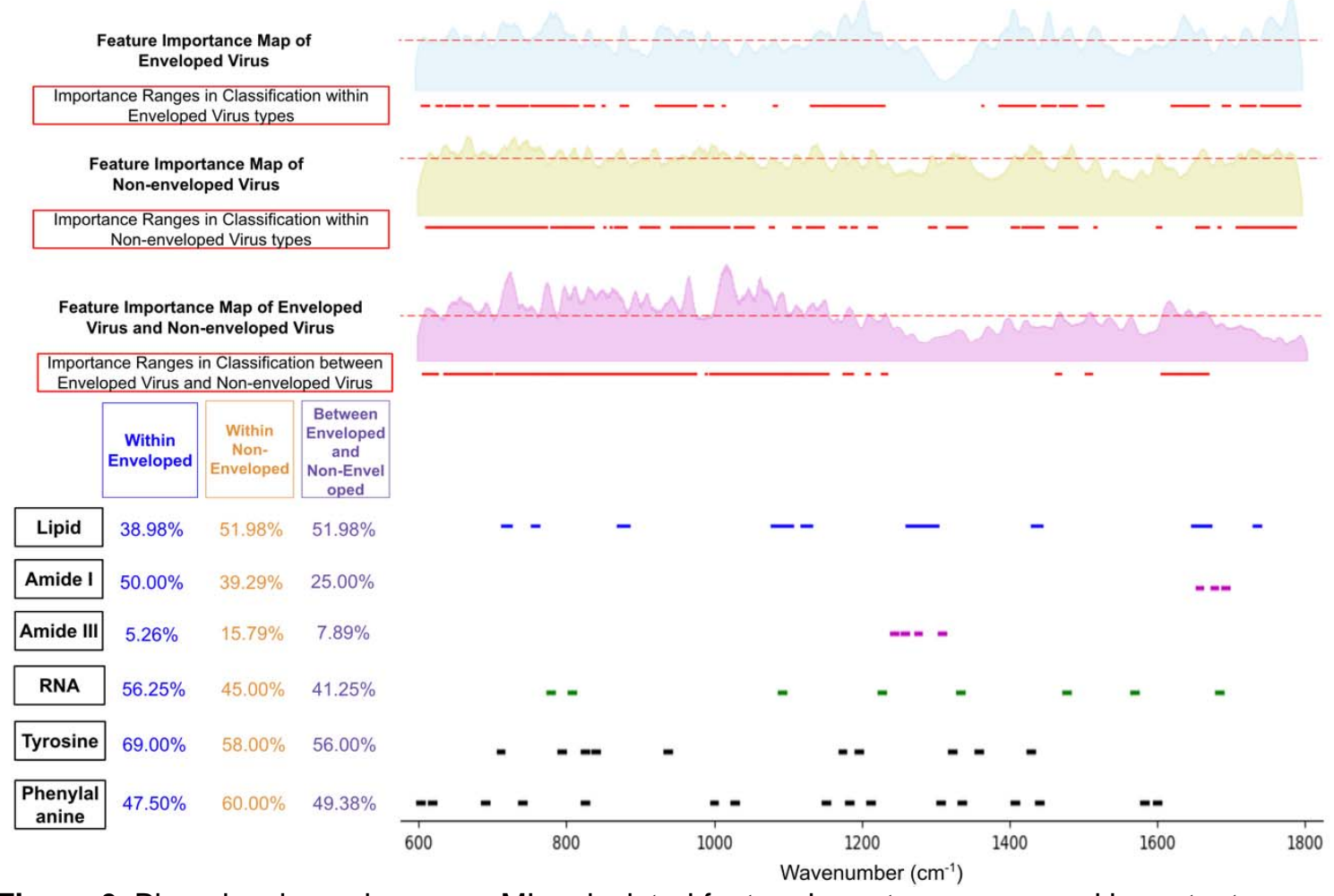

Figure 6. Biomolecule peak ranges, ML-calculated feature importance map, and important Raman ranges (above $40^{\text {th }}$ percentile threshold) for classification experiments: 1 ) Within enveloped virus types (Avian FLUA, IBV Coronavirus, Human FLUA, Human FLUB, RSV); 2) Within non-enveloped virus types (Enterovirus (CVB1, CVB3, EV70, EV71, PV2), Rhino, Reovirus); 3) Between Enveloped and Non-enveloped viruses. Feature importance maps are extracted from intermediate layers of the CNN as described in Fig. 1B. The matching score for each classification experiment is calculated by correlating ML-selected important ranges with each biomolecule's known Raman peak ranges. (See SI Appendix, Fig. S6 for matching scores with more functional groups) 


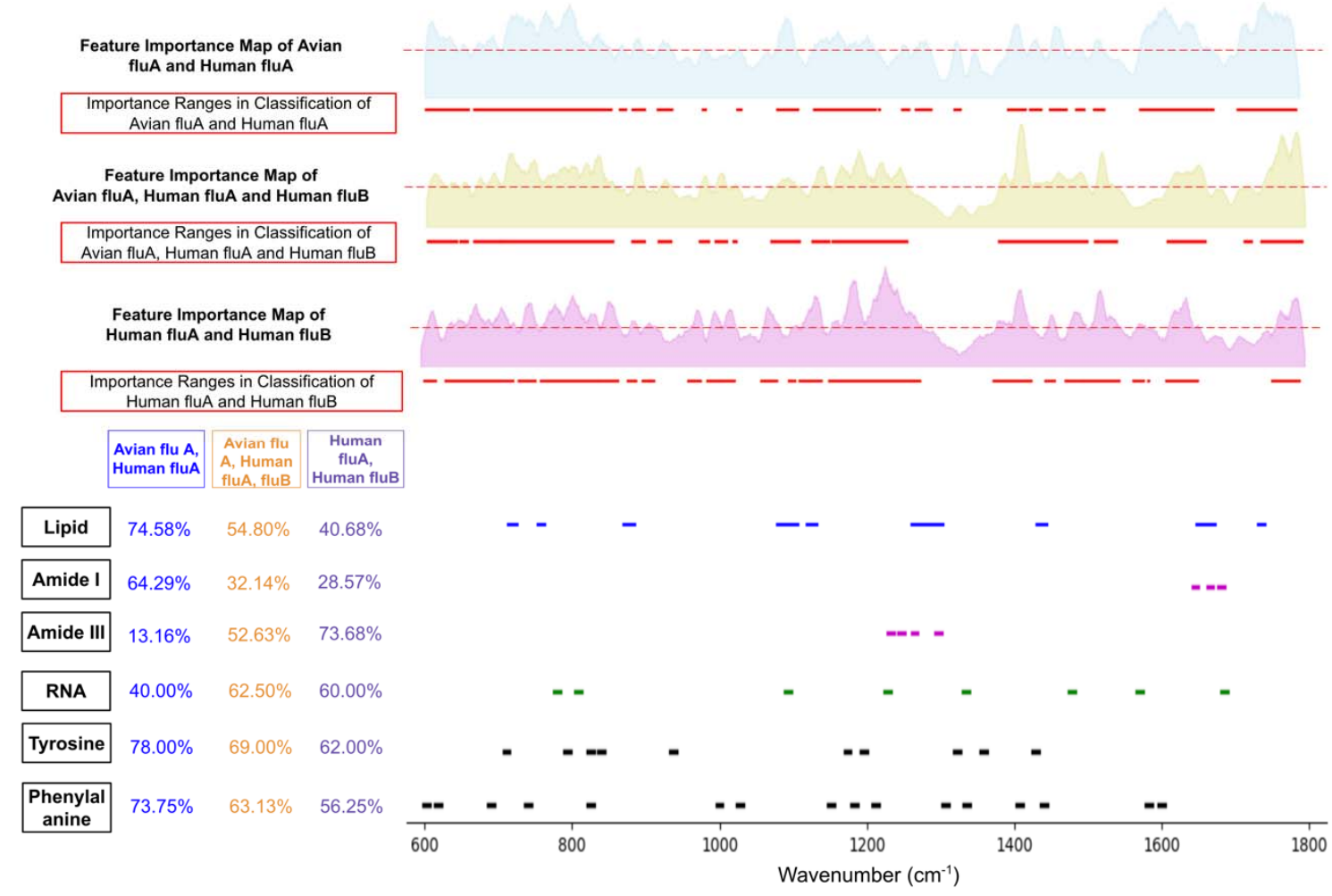

Figure 7. ML-calculated feature importance map, and important Raman ranges for classification experiments: 1) Avian FLUA vs. Human FLUA; 2) Avian FLUA, Human FLUA and Human FLUB; 3) Human FLUA and Human FLUB. (See SI Appendix, Fig. S5 for matching scores with more functional groups). 


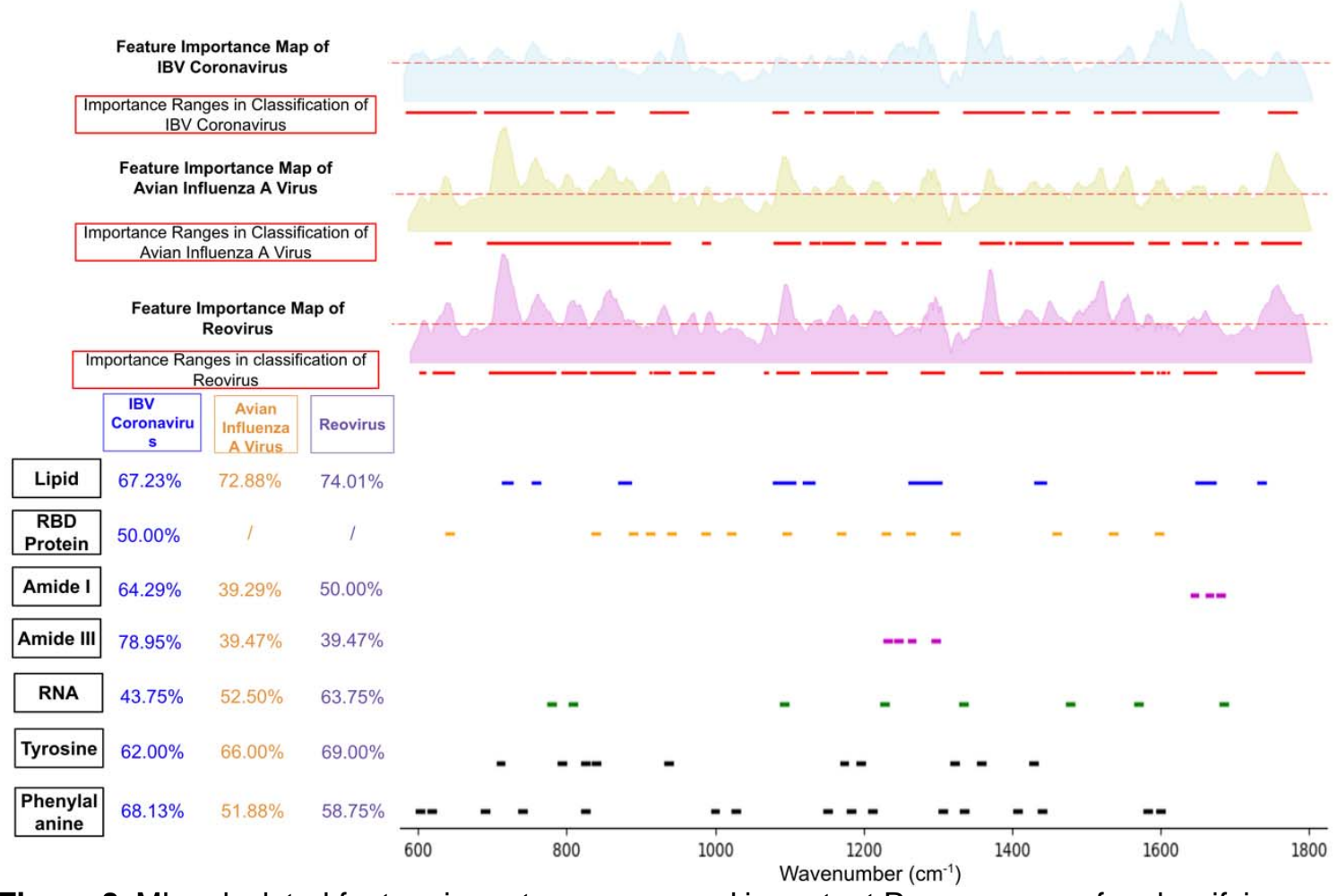

Figure 8. ML-calculated feature importance map, and important Raman ranges for classifying three types of avian viruses: IBV Coronavirus, Avian Influenza A virus and Reovirus. Feature important maps and matching scores are given for each avian virus type. The matching score for RBD Protein only applies when correlating with IBV Coronavirus because RBD Protein is an exclusive biomolecule in IBV. (See SI Appendix, Fig. S1 for matching scores with more functional groups). 